\title{
Sur l'existence de la fonction de Green pour un problème aux limites relatif à une équation différentielle à argument fonctionnel
}

\author{
ANNA SoROLEWSEA (Rzeszów)
}

Résumé. Dans ce travail nous étudions le problème aux limites suivant:

(1)

(2)

$$
\begin{aligned}
& y^{\prime \prime}(t)=f(l) \cdot y(\delta(t)), \quad t_{0}<\delta(t)<t, \quad t \in\left[t_{0}, T\right], \\
& U_{1}\left(y, y^{\prime}\right)=\sum_{\nu=0}^{1}\left[a_{1 v} y^{(\nu)}\left(t_{0}\right)+\beta_{1,} y^{(v)}(T)\right]=0, \\
& U_{2}\left(y, y^{\prime}\right)=\sum_{\nu=0}^{1}\left[a_{2 \nu} y^{(v)}\left(t_{0}\right)+\beta_{2,}, y^{(\nu)}(T)\right]=0 .
\end{aligned}
$$

Pour le problème (1), (2) wous démontrons l'existence de la fonotion de Green. Pour la démonstration nous remplaçons le problème (1), (2) par le problème équivalent suivant:

$$
\begin{gathered}
y_{0}^{\prime}(t)=y_{1}(t), \\
y_{1}^{\prime}(t)=f(t) \cdot y_{0}(\delta(t)), \\
y_{0}\left(t_{0}\right)=k_{0}, \quad y_{1}\left(t_{0}\right)=k_{1}
\end{gathered}
$$

et nous choisissons un point quelconqug $\xi \epsilon\left(t_{0}, T\right)$. Nous construisons ensuite les lignes brisées d'Euler, convonablement modifieses, $\left(L_{0 n}(t, \xi)\right.$ et $\left.L_{1 n}(t, \xi)\right)$ et nous montrons que pour tout $n$ suffisamment grand il existe un systeme de conditions initiales $k_{0}=k_{0}(\xi), k_{1}=k_{1}(\xi)$ tel que les lignes brisées $\bar{L}_{0 n}(t, \xi)$ et $\bar{L}_{1 n}(t, \xi)$ qui leur correspondent satisfont aux conditions (2).

La fonction $\Gamma(t, \xi)$ est ensuite définie comme la limite de la fonction $\bar{L}_{0 n}(t, \xi)$ si $n \rightarrow+\infty$.

La fonction $\Gamma(t, \xi)$ satisfait à la condition $y(t)=\int_{l_{0}}^{T} y(\xi) \cdot \Gamma(t, \xi) d \xi$.

Dans ce travail nous étudions le problème aux limites suivant:

$$
\begin{gathered}
y^{\prime \prime}(t)=f(t) \cdot y(\delta(t)) \\
J_{1}\left(y, y^{\prime}\right)=\sum_{\nu=0}^{1}\left[\alpha_{1}, y^{(\nu)}\left(t_{0}\right)+\beta_{1 \nu} y^{(\nu)}(T)\right]=0 \\
J_{2}\left(y, y^{\prime}\right)=\sum_{\nu=0}^{1}\left[\alpha_{2 \nu} y^{(\nu)}\left(t_{0}\right)+\beta_{2 \nu} y^{(\nu)}(T)\right]=0 .
\end{gathered}
$$


Pour le problème (1), (2) nous admettons les hypothèses suivantes:

$1^{0}$ la fonction $f(t)$ est continue dans l'intervalle $\left[t_{0}, T\right]$,

$2^{\circ} \delta(t)$ est une fonction continue dans l'intervalle $\left[t_{0}, T\right]$ et elle satisfait aux conditions: $\delta\left(t_{0}\right)=t_{0}, t_{0} \leqslant \delta(t) \leqslant t$ pour $t \epsilon\left(t_{0}, T\right]$,

$3^{\circ}$ la solution unique du problème (1), (2) est la solution triviale.

En nous appuyant sur les hypothèses $1^{\circ}, 2^{\circ}, 3^{\circ}$ nous allons démontrer l'existence de la fonction de Green pour le problème (1), (2), la démonstration ayant un caractère effectif.

Dans ce but considérons le problème initial de Cauchy suivant:

$$
\begin{gathered}
y^{\prime \prime}(t)=f(t) \cdot y(\delta(t)), \\
y\left(t_{0}\right)=k_{0}, \quad y^{\prime}\left(t_{0}\right)=k_{1}
\end{gathered}
$$

( $k_{0}$ et $k_{1}$ étant pour le moment des nombres arbitrairement fixés).

Le problème (3), (4) peut être remplacé par le système équivalent d'équations différentielles du premier ordre:

$$
\begin{aligned}
& y_{0}^{\prime}(t)=y_{1}(t), \\
& y_{1}^{\prime}(t)=f(t) \cdot y_{0}(\delta(t))
\end{aligned}
$$

avec les conditions initiales

$$
y_{0}\left(t_{0}\right)=k_{0}, \quad y_{1}\left(t_{0}\right)=k_{1} \text {. }
$$

Choisissons wn point quelconque $\xi \in\left(t_{0}, T\right)$ et divisons chacun des intervalles $\left[t_{0}, \xi\right],[\xi, T]$ en $n$ parties égales par les points $t_{1}, t_{2}, \ldots, t_{2 n-1}$ tels que

$$
t_{0}<t_{1}<\ldots<t_{n}=\xi<t_{n+1}<\ldots<t_{2 n-1}<t_{2 n}=T .
$$

Construisons ensuite les lignes brisées $L_{0 n}(t, \xi)$ (contenue dans le plan $\left(t, y_{0}\right)$ ) et $L_{1 n}(t, \xi)$ (contenue dans le plan $\left.\left(t, y_{1}\right)\right)$. Les ordonnées des sommets consécutifs de ces lignes brisées seront données par les formules:

$$
\begin{aligned}
& y_{0,0}(\xi)=k_{0}, \\
& y_{0 i}(\xi)= \begin{cases}y_{0, i-1}(\xi)+\frac{\xi-t_{0}}{n} y_{1, i-1}(\xi) & \text { pour } i=1,2, \ldots, n, \\
y_{0, i-1}(\xi)+\frac{T-\xi}{n} y_{1, i-1}(\xi) & \text { pour } i=n+1, \ldots, 2 n,\end{cases}
\end{aligned}
$$

(6)

$$
\begin{aligned}
& y_{1,0}(\xi)=k_{1}, \\
& y_{1 i}(\xi)=\left\{\begin{array}{l}
y_{1, i-1}(\xi)+\frac{\xi-t_{0}}{n} f\left(t_{i-1}\right) L_{0 n}\left(\delta\left(t_{i-1}\right), \xi\right) \text { pour } i=1,2, \ldots, n, \\
y_{1, i-1}(\xi)+\frac{T-\xi}{n} f\left(t_{i-1}\right) L_{0 n}\left(\delta\left(t_{i-1}\right), \xi\right) \text { pour } i=n+1, \ldots, 2 n .
\end{array}\right.
\end{aligned}
$$


Nous allons montrer par récurrence que les ordonnées $y_{0 l}, y_{1}$ $(l=0,1, \ldots, 2 n)$ satisfont aux relations:

$$
y_{0 l}(\xi)=a_{0 l}^{0}(\xi) k_{0}+a_{01}^{1}(\xi) k_{1}, \quad y_{1 l}(\xi)=a_{1 l}^{0}(\xi) k_{0}+a_{1 l}^{1}(\xi) k_{1},
$$

oì les coefficients de $k_{0}$ et $k_{1}$ ne dépendent que des fonctions $f(t)$ et $\delta(t)$ ainsi que des points du réseau (5).

Pour $l=0$ les formules (7) ont bien lieu. Supposons-les vraies pour $i \leqslant l-1$. Alors

$$
\begin{aligned}
& y_{0 l}(\xi)=y_{0, l-1}(\xi)+\frac{\xi-t_{0}}{n} y_{1, l-1}(\xi)=\sum_{j=0}^{1}\left(a_{0, l-1}^{j}(\xi)+\frac{\xi-t_{0}}{n} a_{1, l-1}^{j}(\xi)\right) t_{j}, \\
& y_{1 l}(\xi)=y_{1, l-1}(\xi)+\frac{\xi-t_{0}}{n} f\left(t_{l-1}\right) \cdot \underline{L}_{0 n}\left(\delta\left(t_{l-1}\right), \xi\right) \\
& =\sum_{j=0}^{1}\left[a_{1, l-1}^{j}(\xi)+\frac{\xi-t_{0}}{n} f\left(t_{l-1}\right)\left(\gamma \cdot a_{0, p-1}^{j}(\xi)+(1-\gamma) a_{0, p}^{j}(\xi)\right)\right]: k_{j}, \\
& \text { si } \delta\left(t_{l-1}\right) \in\left[t_{p-1}, t_{p}\right), l \leqslant n, \text { avec } \gamma=\frac{t_{p}-\delta\left(t_{l-1}\right)}{t_{p}-t_{p-1}} \text {. }
\end{aligned}
$$

D'une façon analogue on peut montrer que les relations (7) sont VTraies pour $l>n$.

On construit ensuite les lignes brisées $\bar{L}_{0 n}(t, \xi)$ (contenue dans le plan $\left.\left(t, y_{0}\right)\right)$ et $\bar{L}_{1 n}(t, \xi)$ (contenue dans le plan $\left.\left(t, y_{1}\right)\right)$, en profitant encore des points du réseau (5). Les ordonnées des sommets consécutifs de ces lignes brisées seront déterminées par les relations:

$$
\begin{aligned}
\bar{y}_{0 i}(\xi) & =y_{0 i}(\xi) \quad \text { pour } i=0,1, \ldots, n-1, \\
\bar{y}_{0 i}(\xi) & =\bar{y}_{0, i-1}(\xi)+\frac{T-\xi}{n} \bar{y}_{1, i-1}(\xi) \quad \text { pour } i=n, \ldots, 2 n, \\
\bar{y}_{1 i}(\xi) & =y_{1 i}(\xi) \quad \text { powr } i=0,1, \ldots, n-1, \\
\bar{y}_{1 n}(\xi) & =y_{1 n}(\xi)+1, \\
\bar{y}_{1 i}(\xi) & =\bar{y}_{1, i-1}(\xi)+\frac{T-\xi}{n} f\left(t_{i-1}\right) \bar{L}_{0 n}\left(\delta\left(t_{i-1}\right), \xi\right)
\end{aligned}
$$

pour $i=n+1, \ldots, 2 n$. 
De même que dans le cas des relations (7) on peut montrer que (9) $\bar{y}_{0 l}(\xi)=y_{0 l}(\xi)+b_{0 l}(\xi), \quad \bar{y}_{1 l}(\xi)=y_{1 l}(\xi)+b_{1 l}(\xi) \quad(l=0,1, \ldots, 2 n)$, où les nombres $b_{0 l}(\xi)$ et $b_{1 l}(\xi)$ dépendent uniquement des fonctions $f(t)$ et $\delta(t)$ ainsi que des points du réseau (5).

Des relations (7) ot (9) il résulte, en particulier, que

$$
\begin{aligned}
& y_{0,2 n}(\xi)=a_{0,2 n}^{0}(\xi) k_{0}+a_{0,2 n}^{1}(\xi) k_{1} ; \\
& y_{1,2 n}(\xi)=a_{1,2 n}^{0}(\xi) k_{0}+a_{1,2 n}^{1}(\xi) k_{1} ; \\
& \bar{y}_{0,2 n}(\xi)=a_{0,2 n}^{0}(\xi) k_{0}+a_{0,2 n}^{1}(\xi) k_{1}+b_{0,2 n}(\xi) ; \\
& \bar{y}_{1,2 n}(\xi)=a_{1,2 n}^{0}(\xi) k_{0}+a_{1,2 n}^{1}(\xi) k_{1}+b_{1,2 n}(\xi) .
\end{aligned}
$$

Désignons par $L_{0 n}^{\mathrm{I}}(t, \xi), L_{1 n}^{\mathrm{I}}(t, \xi)$ et $L_{0 n}^{\mathrm{II}}(t, \xi), L_{1 n}^{\mathrm{II}}(t, \xi)$ les lignes brisées, définies précédemment (voir (6)), liées respectivement aux systèmes de conditions initiales $\left(k_{0}, k_{1}\right)=(1,0)$ et $\left(k_{0}, k_{1}\right)=(0,1)$, et désignons parr $L_{0 n}^{\mathrm{III}}(t, \xi), L_{1 n}^{\mathrm{III}}(t, \xi)$ les lignes brisées définies par les conditions (8) et correspondant aux conditions initiales $\left(k_{0}, k_{1}\right)=(0,0)$. Les coefficients dans les relations (10) satisfont aux conditions

$$
\begin{array}{lll}
a_{0,2 n}^{0}(\xi)=L_{0 n}^{\mathrm{I}}(T, \xi), & a_{1,2 n}^{0}(\xi)=L_{1 n}^{\mathrm{I}}(T, \xi), & b_{0,2 n}(\xi)=\bar{L}^{\mathrm{III}}(T, \xi), \\
a_{1,2 n}^{1}(\xi)=L_{0 n}^{\mathrm{II}}(T, \xi), & a_{1,2 n}^{\mathrm{I}}(\xi)=L_{1 n}^{\mathrm{II}}(T, \xi), & b_{1,2 n}(\xi)=\bar{L}_{1 n}^{\mathrm{III}}(T, \xi) .
\end{array}
$$

De plus, les théorèmes d'existence et d'unicité de la solution du problème initial de Cauchy pour les équations différentielles à argument retardé [2], [3] impliquent 1'existence des limites $\lim _{n \rightarrow \infty} b_{0,2 n}(\xi), \lim _{n \rightarrow \infty} b_{1,2 n}(\xi)$, ainsi que des limites:

$$
\begin{array}{ll}
\lim _{n \rightarrow \infty} a_{0,2 n}^{0}(\xi)=y_{0}^{\mathrm{I}}(T), & \lim _{n \rightarrow \infty} a_{1,2 n}^{0}(\xi)=y_{1}^{\mathrm{I}}(T), \\
\lim _{n \rightarrow \infty} a_{0,2 n}^{1}(\xi)=y_{0}^{\mathrm{II}}(T), & \lim _{n \rightarrow \infty} a_{1,2 n}^{1}(\xi)=y_{1}^{\mathrm{II}}(T),
\end{array}
$$

où $\left\{y_{0}^{\mathrm{I}}(t), y_{1}^{\mathrm{I}}(t)\right\},\left\{y_{0}^{\mathrm{II}}(t), y_{1}^{\mathrm{II}}(t)\right\}$ désignent les solutions du problème $\left(3^{\prime}\right)$, $\left(4^{\prime}\right)$ qui correspondent respectivement aux systèmes de conditions initiales $\{1,0\}$ et $\{0,1\}$.

Nous allons montrer que pour tout $n$ suffisamment grand il existe exactement un systeme de conditions initiales $\left\{k_{0 n}, k_{1 n}\right\}$ tel que les lignes brisées $\bar{L}_{0 n}(t, \xi)$ et $\bar{L}_{1 n}(t, \xi)$ qui leur correspondent satisfont aux conditions:

$$
U_{1}\left(\bar{L}_{0 n}(t, \xi), \bar{L}_{1 n}(t, \xi)\right)=0, \quad V_{2}\left(\bar{L}_{0 n}(t, \xi), \bar{L}_{1 n}(t, \xi)\right)=0 .
$$

Les fonctions $\sigma_{1}$ et $U_{2}$ étant linéaires ( $\nabla$. (2)), on peut, en tenant compte des relations (9) et (10), mettre les conditions (13) sous la forme

$$
\begin{aligned}
& U_{1}\left(L_{0 n}(t, \xi), L_{1 n}(t, \xi)\right)=A_{1 n}^{0}(\xi) k_{0}+A_{1 n}^{1}(\xi) k_{1}=B_{1 n}(\xi), \\
& U_{2}\left(L_{0 n}(t, \xi), L_{1 n}(t, \xi)\right)=A_{2 n}^{0}(\xi) k_{0}+A_{2 n}^{1}(\xi) k_{1}=B_{2 n}(\xi) .
\end{aligned}
$$


Les coefficients dans les relations $\left(13^{\prime}\right)$ dépendent continûment des coefficients qui figurent dans les formules (10); de plus, les remarques précédentes avec les conditions (12) entrainent l'existence des limites finies

$$
\begin{gathered}
\lim _{n \rightarrow \infty} \operatorname{det}\left[\begin{array}{cc}
A_{1 n}^{0}(\xi) & A_{1 n}^{1}(\xi) \\
A_{2 n}^{0}(\xi) & A_{2 n}^{1}(\xi)
\end{array}\right], \quad \lim _{n \rightarrow \infty} \operatorname{det}\left[\begin{array}{ll}
B_{1 n}(\xi) & A_{1 n}^{1}(\xi) \\
B_{2 n}(\xi) & A_{2 n}^{1}(\xi)
\end{array}\right], \\
\lim _{n \rightarrow \infty} \operatorname{det}\left[\begin{array}{ll}
A_{1 n}^{0}(\xi) & B_{1 n}(\xi) \\
A_{2 n}^{0}(\xi) & B_{2 n}(\xi)
\end{array}\right]
\end{gathered}
$$

La première des limites (14) satisfait, en outre, à la condition suivante:

$$
\begin{gathered}
\lim _{n \rightarrow \infty} \operatorname{det}\left[\begin{array}{ll}
A_{1 n}^{0}(\xi) & A_{1 n}^{\mathrm{I}}(\xi) \\
A_{2 n}^{\mathrm{O}}(\xi) & A_{2 n}^{\mathrm{I}}(\xi)
\end{array}\right] \\
\quad=\lim _{n \rightarrow \infty} \operatorname{det}\left[\begin{array}{l}
U_{1}\left(L_{0 n}^{\mathrm{I}}(t, \xi) L_{1 n}^{\mathrm{I}}(t, \xi)\right) U_{1}\left(L_{0 n}^{\mathrm{II}}(t, \xi), L_{1 n}^{\mathrm{II}}(t, \xi)\right) \\
U_{2}\left(L_{0 n}^{\mathrm{I}}(t, \xi) L_{1 n}^{\mathrm{I}}(t, \xi)\right) U_{2}\left(L_{0 n}^{\mathrm{II}}(t, \xi), L_{1 n}^{\mathrm{II}}(t, \xi)\right)
\end{array}\right] \\
\quad=\operatorname{det}\left[\begin{array}{ll}
U_{1}\left(y_{0}^{\mathrm{I}}, y_{1}^{\mathrm{I}}\right) & U_{1}\left(y_{0}^{\mathrm{II}}, y_{1}^{\mathrm{II}}\right) \\
U_{2}\left(y_{0}^{\mathrm{I}}, y_{1}^{\mathrm{I}}\right) & U_{2}\left(y_{0}^{\mathrm{II}}, y_{1}^{\mathrm{II}}\right)
\end{array}\right] \neq 0 .
\end{gathered}
$$

La condition (15) est, dans sa dernière partie, une conséquence de l'hypothèse $3^{\circ}$. II en résulte pour $n$ suffisamment grand l'existence d'un seul système de nombres $\left\{k_{0 n}(\xi), k_{1 n}(\xi)\right\}$ satisfaisant aux conditions (13') ainsi quo l'existence des limites finies $\lim k_{0 n}(\xi), \lim k_{1 n}(\xi)$. Nous désignerons désormais par $\bar{L}_{0 n}(t, \xi)$ et $\bar{L}_{1 n}(t, \xi)$ les lignes brisées qui vérifient les conditions (13), c'est-à-dire celles qui correspondent aux systèmes de conditions initiales satisfaisant à $\left(13^{\prime}\right)$.

Définissons maintenant la fonction $\Gamma(t, \xi)$ comme il suit:

$$
\begin{aligned}
& \Gamma(t, \xi)=\lim _{n \rightarrow \infty} \bar{L}_{0 n}(t, \xi) \quad \text { pour } \xi \neq t_{0}, \xi \neq T, \\
& \Gamma\left(t, t_{0}\right)=\lim \Gamma(t, \xi), \\
& \xi(t, T)=\lim \Gamma(t, \xi) .
\end{aligned}
$$

On constate sans peine que la fonction définie par les formules (16) vérifie pour tout $\xi \in\left[t_{0}, T\right]$ les conditions suivantes ( $\nabla$. [2]):

1. La fonction $\Gamma(t, \xi)$ est continue pour tout $t \in\left[t_{0}, T\right]$.

2. La fonction $\Gamma_{t}^{\prime}(t, \xi)$ est continue pour tout $t \in\left[t_{0}, T\right]$, sauf au point $t=\xi$, où elle admet un saut égal à 1 , c'est-à-dire $\Gamma_{l}^{\prime}(\xi+0, \xi)-\Gamma_{t}^{\prime}(\xi-$ $-0, \xi)=1$.

3. $y=\Gamma(t, \xi)$ satisfait pour tout $t \epsilon\left[t_{0}, T\right]$, excepté au point $t=\xi$, à l'équation différentielle (1).

4. $y=\Gamma(t, \xi)$ vérifie les conditions aux limites (2).

Par conséquent on peut démontrer, de même que dans le cas d'une équation différentielle sans argument dévié [1], que la solution unique 
du problème aux limites semi-homogène

$$
y^{\prime \prime}(t)=f(t) y(\delta(t))+g(t)
$$

$\left(g(t)\right.$ est une fonction continue dans l'intervalle $\left.\left[t_{0}, T\right]\right)$,

$$
\begin{aligned}
& U_{1}\left(y, y^{\prime}\right)=0, \\
& U_{2}\left(y, y^{\prime}\right)=0
\end{aligned}
$$

est de la forme

$$
y(t)=\int_{t_{0}}^{T} g(\xi) \Gamma(t, . \xi) d \xi .
$$

En terminant, observons que par un procédé analogue à celui qui a été utilisé plus haut, on peut construire la fonction de Green dans un cas plus général, à savoir pour l'équation différentielle de la forme

$$
y^{(n)}(t)=\sum_{p=0}^{n-2} f_{v}(t) y^{(v)}\left(\delta_{(v)}(t)\right)+f_{n-1}(t) y^{(n-1)}(t)
$$

arec les conditions aux limites

$$
\delta_{\mu}\left(y, y^{\prime}, \ldots, y^{(n-1)}\right)=\sum_{\nu=0}^{n-1}\left[\alpha_{\mu \nu} y^{(v)}\left(t_{0}\right)+\beta_{\mu \nu} y^{(\nu)}(T)\right] \quad(\mu=1,2, \ldots, n)
$$

\section{Références}

[1] E. Kamke, Differentialgleichungen reeller Funktionen, Leipzig 1956.

[2] А. Д. Мыпाис, Линейные дифференчиальные уравнения с аапаздываюиция араументом, Москви-Ленинград 1951,

[3] K. Zima, O jednoznaczności rozwiazania problemu Oauohy'ego dla równasi różniczkowyoh z presunietym argumentem, Zeszyty Naukowe WSP w Katowicach, SM, 5 (1966), p. 75-81. 